\title{
Sharp lower bounds for the asymptotic entropy of symmetric random walks
}

\author{
Sébastien Gouëzel, Frédéric Mathéus, and François Maucourant
}

\begin{abstract}
The entropy, the spectral radius and the drift are important numerical quantities associated to random walks on countable groups. We prove sharp inequalities relating those quantities for walks with a finite second moment, improving upon previous results of Avez, Varopoulos, Carne, Ledrappier. We also deduce inequalities between these quantities and the volume growth of the group. Finally, we show that the equality case in our inequality is rather rigid.
\end{abstract}

Mathematics Subject Classification (2010). 05C81, 60B15, 60J50.

Keywords. Random walk, countable group, entropy, spectral radius, drift, volume growth, Poisson boundary.

\section{Introduction and main results}

Let $\Gamma$ be a countable group and $\mu$ a probability measure on $\Gamma$. The right random walk associated with the pair $(\Gamma, \mu)$ is the Markov chain on $\Gamma$ whose transition probabilities are defined by $p(x, y)=\mu\left(x^{-1} y\right)$. A realization of the random walk starting from the identity is given by $X_{0}=e$ and $X_{n}=\gamma_{1} \ldots \gamma_{n}$ where $\left(\gamma_{i}\right)_{i}$ is an independent sequence of $\Gamma$-valued $\mu$-distributed random variables. The law of $X_{n}$ is the $n$-fold convolution $\mu^{* n}$ of $\mu$.

Let $|\cdot|=\operatorname{dist}(\cdot, e)$ denote the distance to the identity, for a proper left- $\Gamma$-invariant distance $\operatorname{dist}(\cdot, \cdot)$ on $\Gamma$ (in examples, we will choose implicitly the word length with respect to a finite symmetric set of generators $S$ ). Several numerical quantities were introduced to describe the asymptotic behavior of $X_{n}$. The asymptotic entropy $h$, the spectral radius $\rho$ and the drift (or rate of escape) $\ell$ of the random 
walk with respect to $|\cdot|$ are defined by

$$
\begin{aligned}
& h=\lim _{n}-\frac{1}{n} \sum_{g} \mu^{* n}(g) \log \mu^{* n}(g), \\
& \rho=\limsup _{n} \sqrt[n]{\mu^{* n}(e)} \leqslant 1
\end{aligned}
$$

and

$$
\ell=\lim _{n} \frac{1}{n} \sum_{g}|g| \mu^{* n}(g)
$$

The asymptotic entropy is well-defined if the entropy

$$
H(\mu)=-\sum_{g \in \Gamma} \mu(g) \log \mu(g)
$$

is finite. No assumption on the measure $\mu$ is required to define the spectral radius $\rho$. The drift $\ell$ is well-defined if $\mu$ has finite first moment. Note that if the cardinality of the balls $B(e, n)$ grows at most exponentially, then finiteness of the first moment implies finiteness of the entropy, see [7, 14].

Assume that $\mu$ is supported on a finite set of generators $S$, and that dist is the corresponding word distance. When the set $S$ has $2 d$ elements, the drift is bounded by the drift of the simple random walk in a regular tree with valence $2 d$, i.e., $1-1 / d$. However, if there are a lot of relations in the group, the walk is more likely to come back closer to the identity, and one would expect a smaller drift. In this direction, it is more relevant to consider the volume growth $v=\lim _{n} \frac{1}{n} \log \# B(e, n)$ of $\Gamma$ with respect to $S$ rather than merely the number of generators: one may expect that a bound on $v$ implies a bound on the drift, of the form $\ell \leqslant f(v)$ for some function $f$ taking values in $[0,1)$. Such an inequality is surprisingly hard to prove directly. Our first result answers this question, for an explicit function $f$. A similar discussion holds for the spectral radius (one can bound $\rho$ from below using the number of generators, by $\sqrt{2 d-1} / d$, see Kesten [16], but bounds involving $v$ are harder to come with).

Our inequalities hold for measures with finite second moment; throughout the paper, we will write

$$
M_{2}(\mu):=\left(\sum_{g}|g|^{2} \mu(g)\right)^{1 / 2}
$$

for the $\ell^{2}$-norm with respect to the measure $\mu$ of the distance to the identity. 
Theorem 1.1. Let $\Gamma$ be a countable group with a proper left-invariant distance, such that $v=\liminf _{n} \frac{1}{n} \log \# B(e, n)$ is finite. Let $\mu$ be a symmetric probability measure with a finite second moment on $\Gamma$. Denote by

$$
\tilde{\ell}=\ell / M_{2}(\mu) \text { and } \tilde{v}=M_{2}(\mu) v
$$

the drift and the growth for the distance $\widetilde{\operatorname{dist}}(g, h)=\operatorname{dist}(g, h) / M_{2}(\mu)$. The following inequalities hold:

$$
\tilde{\ell} \leqslant \tanh (\tilde{v} / 2), \quad h \leqslant \tilde{v} \tanh (\tilde{v} / 2), \quad \rho \geqslant 1 / \cosh (\tilde{v} / 2) .
$$

These inequalities are consequences of other inequalities relating $h$ to $\ell$ and $\rho$ :

Theorem 1.2. Let $\mu$ be a symmetric probability measure with finite entropy on a countable group $\Gamma$ with a proper left-invariant distance. Then

$$
2 \sqrt{1-\rho^{2}} \operatorname{artanh} \sqrt{1-\rho^{2}} \leqslant h .
$$

Moreover, if $\mu$ has a finite second moment,

$$
2 \tilde{\ell} \operatorname{artanh} \tilde{\ell} \leqslant h
$$

where $\tilde{\ell}=\ell / M_{2}(\mu)$.

The first theorem is a consequence of the second one:

Proof of Theorem 1.1 using Theorem 1.2. The entropy satisfies the so-called "fundamental inequality" $h \leqslant \ell v=\tilde{\ell} \tilde{v}$, by [12]. Since $2 \tilde{\ell} \operatorname{artanh} \tilde{\ell} \leqslant h$ by (1.2), this yields $2 \operatorname{artanh}(\tilde{\ell}) \leqslant \tilde{v}$, hence $\tilde{\ell} \leqslant \tanh (\tilde{v} / 2)$. Since $h \leqslant \tilde{\ell} \tilde{v}$, we deduce that $h \leqslant \tilde{v} \tanh (\tilde{v} / 2)$. Last, we remark that $r=1 / \cosh (\tilde{v} / 2)$ satisfies

$$
2 \sqrt{1-r^{2}} \operatorname{artanh} \sqrt{1-r^{2}}=2 \tanh (\tilde{v} / 2) \operatorname{artanh}(\tanh (\tilde{v} / 2))=\tilde{v} \tanh (\tilde{v} / 2) .
$$

We have already proved that this is larger than or equal to $h$. Together with (1.1) and the fact that $t \mapsto 2 \sqrt{1-t^{2}} \operatorname{artanh} \sqrt{1-t^{2}}$ is non-increasing, this gives $\rho \geqslant r$, as claimed.

The inequalities of Theorem 1.2 have several predecessors. The first lower bound for the asymptotic entropy is due to A. Avez [2], who proved that $h \geqslant-2 \log \rho$. More recently, Ledrappier [22] showed that $h \geqslant 4(1-\rho)$. Those two inequalities are not comparable, Ledrappier's being stronger for $\rho$ close to 1 but weaker for $\rho$ close to 0 . The inequality (1.1) is a common strengthening of both inequalities of Avez and Ledrappier, since the left-hand side of (1.1) is larger 
than $\max (-2 \log \rho, 4(1-\rho))$ (and asymptotic to $-2 \log \rho$ when $\rho$ tends to 0 , and to $4(1-\rho)$ when $\rho$ tends to 1$)$. This statement may not be obvious from the formula (1.1), but it follows readily from the analysis of this function that we will have to do later on (see in particular Lemma 3.4).

Lower bounds for the asymptotic entropy $h$ involving the drift $\ell$ were also considered. Varopoulos [27] and Carne [6] proved that

$$
\mu^{* n}(g) \leqslant 2 \exp \left[-\frac{|g|^{2}}{2 n k^{2}}\right] \text { for all } g \in \Gamma,
$$

where $k$ is the radius of the smallest ball containing the support of $\mu$. The consequence for $h$ and $\ell$ becomes $h \geqslant \ell^{2} / 2 k^{2}$.

Theorem 1.2 improves this inequality in several ways: it replaces $k$ by $M_{2}(\mu)$ (allowing measures with infinite support), it replaces the square function with the better function $2 x \operatorname{artanh}(x)$ (which is $x^{2}+o\left(x^{2}\right)$ at 0 , and strictly larger than $x^{2}$ away from 0 ), and it gains a multiplicative factor of 2 .

Recently, A. Erschler and A. Karlsson proved in [10] that $h \geqslant \ell^{2} / C(\mu)$ still holds for symmetric probability measures with finite second moment giving nonzero probability to the identity, where $C(\mu)$ depends on $\mu$ (the main dependency is on $\mu(e)>0$ and on $\left.M_{2}(\mu)<\infty\right)$.

Theorem 1.2 owes a lot to [22] and [10]: our investigations started when we tried to understand and sharpen the arguments in those two papers. The proofs in these articles are given inside the group, studying the random walk at finite time (or a poissonized version of the random walk in [22]). It turns out that Theorem 1.2 can be proved following the same strategy. However, an (essentially equivalent) proof can also be given using various boundaries (the Poisson boundary for the inequality involving $\rho$, the horocycle boundary for the inequality involving $\ell$ ). This proof has the advantage of avoiding limits completely, making it possible to characterize the equality case in our inequalities (see Proposition 2.4 below). Therefore, we will concentrate mainly on the proof using boundaries: at the beginning of Section 3, we will quickly sketch the proofs inside the group, without giving all the details, and the rest of Section 3 will be devoted to a complete proof using boundaries. Before this, Section 2 is devoted to more examples and comments.

Let us stress that inequalities similar to the results of Theorem 1.2 have been known for a longer time for Brownian motion on cocompact Riemannian manifolds (see for instance [13, 21, 24]): infinitesimal inequalities are available and make for a simpler result. 


\section{Examples and comments}

Let us first note that the conclusion of Theorem 1.2 does not hold any more if the measure $\mu$ is not symmetric. For instance, for the random walk on $\mathbb{Z}$ given by $\mu=p \delta_{-1}+(1-p) \delta_{+1}$, one has $h=0$ while $\ell=|2 p-1|$ and $\rho=2 \sqrt{p(1-p)}$. When $p \neq 1 / 2$, one gets $\ell>0$ and $\rho<1$, hence Theorem 1.2 does not hold in this case.

Example 2.1. Let $\Gamma=\mathbb{F}\left(a_{1}, \ldots, a_{d}\right)$ be the free non-abelian group over $\left\{a_{1}, \ldots, a_{d}\right\}$, with its usual word distance. We consider the simple random walk on $\Gamma$, i.e., we take for $\mu$ the uniform measure on $S=\left\{a_{1}, \ldots, a_{d}\right\}^{ \pm 1}$. In this case, one can easily compute all the quantities involved in Theorems 1.1 and 1.2. Indeed, one has

- $\ell=1-1 / d$, since at each step away from the identity there is probability $1-1 /(2 d)$ to go further to infinity, and $1 /(2 d)$ to come back.

- $\rho=\frac{\sqrt{2 d-1}}{d}$ since the number of words back to the identity at time $2 n$ has a generating series $\frac{d \sqrt{1-4 z^{2}(2 d-1)}-d+1}{1-4 d^{2} z^{2}}$, with first singularity at $z=1 /(2 \sqrt{2 d-1})$, see [30, Lemma 1.24].

- $h=(1-1 / d) \log (2 d-1)$. This follows for instance from the description of the Poisson boundary as the set of infinite reduced words $b_{0} b_{1} \ldots$, with the measure $v$ giving mass $1 /\left(2 d(2 d-1)^{n-1}\right)$ to any cylinder of length $n$, and from the formula (3.5) below giving the entropy as an integral over the Poisson boundary of the logarithm of the Radon-Nikodym derivative of the group action. See [22] or the proof of Corollary 2.5 for more details.

- $v=\log (2 d-1)$. Indeed, the sphere of radius $n$ has cardinality $2 d(2 d-1)^{n-1}$.

It follows that, in this case, all the inequalities in Theorems 1.1 and 1.2 are equalities. This shows in particular that the inequalities of those theorems are sharp for infinitely many values of the entropy.

Example 2.2. From the free group, one can construct other examples where equality holds in Theorem 1.2. For instance, let $H$ be a finite group and let $\mathbb{F}$ be a free group on finitely many generators $\left\{a_{1}, \ldots, a_{d}\right\}$. In $\Gamma=H \times \mathbb{F}$, consider the generating set $S=\left\{\left(x, a_{i}^{ \pm 1}\right): x \in H, i \in\{1, \ldots, d\}\right\}$. The simple random walk on $(\Gamma, S)$ projects to the simple random walk on the free factor $\mathbb{F}$, and those random walks have the same drift, entropy and spectral radius. Since equality holds in Theorem 1.2 for $\mathrm{F}$, it follows that is also holds for $(\Gamma, S)$. 
More generally, consider an exact sequence

$$
1 \longrightarrow H \longrightarrow \Gamma \longrightarrow \mathrm{F} \longrightarrow 1
$$

where $\mathbb{F}$ is a group whose Cayley graph with respect to some generating system is a tree, and a probability measure on the set of generators of $\Gamma$ that projects to the uniform measure on the generators of $\mathbb{F}$. If the drift, entropy and spectral radius of the random walk on $\Gamma$ are the same as on $\mathbb{F}$ (this is for instance the case if $H$ has subexponential growth), then equality holds in Theorem 1.2 for the random walk on $\Gamma$. Concretely, one may consider for instance any semi-direct product

$$
\Gamma=\mathbb{Z}^{k} \rtimes \mathbb{F}
$$

where $\mathbb{F}$ is a free subgroup of $\mathrm{GL}(k, \mathbb{Z})$. For another example, let

$$
\Gamma^{\prime}=\mathbb{Z} \imath \mathbb{Z} / 3 \mathbb{Z}
$$

with its standard set of generators $S^{\prime}$, let

$$
\Gamma=\Gamma^{\prime} \times \mathbb{F}
$$

and let

$$
S=\left\{\left(x, a_{i}^{ \pm 1}\right): x \in S^{\prime}, i \in\{1, \ldots, d\}\right\}
$$

(this set generates $\Gamma$ since we use $\mathbb{Z} / 3 \mathbb{Z}$ - with $\mathbb{Z} / 2 \mathbb{Z}$ instead, it would generate an index two subgroup of $\Gamma$ ). Since the simple random walk on $\Gamma^{\prime}$ has zero entropy and drift (see [19]), equality in Theorem 1.2 holds for the simple random walk on $\Gamma$. This example is interesting since the volume growth $v$ of $\Gamma$ is strictly larger than the volume growth in the free group as $\Gamma^{\prime}$ has exponential growth. Hence, $h<\ell v$, showing that equality in Theorem 1.2 does not imply equality in the fundamental inequality. There is no implication in the other direction either, see the discussion after Corollary 2.5.

We conjecture (but are unable to prove) that the above situation (2.1) is the only case where equality holds in Theorem 1.2. Partial results in this direction are given in Corollaries 2.5 and 2.6.

Example 2.3. Assume that $\Gamma$ is the fundamental group of a closed compact surface of genus 2. Consider the following presentation of $\Gamma$ :

$$
\Gamma=\left\langle a_{1}, a_{2}, b_{1}, b_{2}:\left[a_{1}, b_{1}\right]\left[a_{2}, b_{2}\right]=1\right\rangle .
$$

The growth $v$ of $\Gamma$ with respect to the generating set $S=\left\{a_{1}, a_{2}, b_{1}, b_{2}\right\}^{ \pm 1}$ is explicitly known. Following Cannon (see [8, § VI.A.8]), it is the logarithm of an algebraic number, and its value is $v=1.9430254 \ldots$. Let $\mu$ be any symmetric probability measure on $S$. Theorem 1.1 gives

$$
\ell \leqslant 0.749368278, \quad h \leqslant 1.456041598, \quad \rho \geqslant 0.66215344 .
$$


This is better than the naive estimates obtained using only the number of generators, by comparing to the free group, giving $\ell \leqslant 0.75$ and $h \leqslant 1.45944$ and $\rho \geqslant 0.66143$. Note that the gain is not very important, but this is not surprising since $\Gamma$ is very close to being free (the growth in the corresponding free group is $\log (7)=1.945910 \ldots$, close to $v$ up to $3 \cdot 10^{-3}$ ).

Assume now that $\mu$ is the uniform measure on $S$. The best known estimates on $\rho$ are $0.662420 \leqslant \rho \leqslant 0.662816$ (see [3] and [26]). It follows that our bound for $\rho$, although worse than Bartholdi's, is precise up to $7.10^{-3}$, while the bound using the number of generators is precise up to $14.10^{-3}$, i.e., twice worse. Using Nagnibeda's upper bound for $\rho$, the inequality (1.1) estimating $h$ in terms of $\rho$ gives $h \geqslant 1.452903618$. Since $\ell \geqslant h / v$, we also have $\ell \geqslant 0.747753281$. This proves that the upper bounds we get for $\ell$ and $h$ are precise up to $2 \cdot 10^{-3}$ and $4.10^{-3}$, to be compared with the bounds using only the number of generators that are precise up to $2.10^{-3}$ and $7.10^{-3}$ : the gain is very small for $\ell$, more significant for $h$.

Let $\Gamma$ be a countable group, and let $\mu$ be a symmetric probability measure on $\Gamma$ whose support generates $\Gamma$, with finite entropy. A $(\Gamma, \mu)$-space is a probability space $(\mathcal{B}, \nu)$ endowed with a $\Gamma$-action, such that the probability $\nu$ is $\mu$-stationary, i.e.,

$$
v=\mu * v \stackrel{\text { def }}{=} \sum_{\gamma \in \Gamma} \mu(\gamma) \gamma_{*} \nu .
$$

In particular, $\gamma_{*} v$ is absolutely continuous with respect to $\nu$, for every $\gamma$ in the subgroup generated by the support of $\mu$, which we assume to coincide with $\Gamma$.

A particularly interesting $(\Gamma, \mu)$-space is its Poisson boundary, that we will denote by $\left(\mathcal{B}_{0}, v_{0}\right)$ : it is the unique $(\Gamma, \mu)$-space parameterizing harmonic functions. Equivalently, it can be seen as the exit boundary of the random walk on the group, made of the events that only depend on the tails of infinite trajectories of the random walk (see [19] for the equivalence and for several other definitions).

The Poisson boundary will play an important role in the proof of Theorem 1.2. It will follow from the proof that the equality case in this theorem implies a rigid behavior of the Poisson boundary:

Proposition 2.4. On a countable group $\Gamma$ with a proper left-invariant distance, consider a symmetric probability measure $\mu$ with finite second moment. Assume that one of the inequalities of Theorem 1.2 is an equality. Then, on the Poisson boundary $\left(\mathcal{B}_{0}, \nu_{0}\right)$ of $(\Gamma, \mu)$, the Radon-Nikodym derivative $\frac{\mathrm{d} \gamma_{*}^{-1} v_{0}}{\mathrm{~d} \nu_{0}}(\xi)$ takes only two values $e^{\alpha}$ and $e^{-\alpha}, \mu \otimes v_{0}$ almost surely. 
There can be no converse to this proposition for the inequality (1.2) involving the distance, since the conclusion of the proposition does not involve the distance. For instance, consider in the free group on two generators $a$ and $b$ a family of distances $d_{\varepsilon}$ giving weight 1 to $a$ and $\varepsilon$ to $b$. If $\mu$ is the uniform measure on the generators, then equality holds in Theorem 1.2 for $d_{1}$, so that the conclusion of Proposition 2.4 holds. On the other hand, the inequality (1.2) is strict for $\tilde{\ell}$ defined using $d_{\varepsilon}$, if $\varepsilon \neq 1$ (one gets $\tilde{\ell}_{\varepsilon}=(1+\varepsilon) / \sqrt{8\left(1+\varepsilon^{2}\right)}<1 / 2=\tilde{\ell}_{1}$ ). We do not know if there is a converse to Proposition 2.4 regarding the inequality (1.1) about the spectral radius.

Proposition 2.4 (the proof of which is given at the end of Section 3) makes it possible to describe precisely some situations where equality can or cannot occur. We should stress that this is very different from the fundamental inequality $h \leqslant \ell v$, where equality is much more difficult to characterize (see [23] for the free group, [25] when $\Gamma$ is a free product of finite groups, or [4] for several characterizations of the equality in terms of quasi-conformal measures on the boundary when $\Gamma$ is a word-hyperbolic group).

Note that, in the previous proposition (and in the corollaries below), the choice of the distance is not important: if there is equality in (1.2) for any proper leftinvariant distance, then the conclusions of Proposition 2.4 hold.

Corollary 2.5. Assume that $\Gamma=\Gamma_{1} * \cdots * \Gamma_{q}(q \geqslant 2)$ is a free product of nontrivial finitely generated groups $\Gamma_{i}$ with finite generating sets $S_{i}$. We exclude the peculiar case $\Gamma=\mathbb{Z} / 2 * \mathbb{Z} / 2$. Let $\mu$ be a symmetric probability measure with support equal to $S=\bigsqcup S_{i}$. Assume that one of the inequalities of Theorem 1.2 is an equality. Then the Cayley graph of each $\Gamma_{i}$ with respect to $S_{i}$ is a regular tree (i.e., $\Gamma_{i}$ is a free product of finitely many factors $\mathbb{Z}$ and $\mathbb{Z} / 2 \mathbb{Z}$ ), the Cayley graph of $\Gamma$ with respect to $S$ is also a regular tree, and $\mu$ is the uniform measure on $S$.

For instance, consider the modular group

$$
\Gamma=\mathbb{Z} / 2 \mathbb{Z} * \mathbb{Z} / 3 \mathbb{Z}=\{1, a\} *\left\{1, b, b^{2}\right\}
$$

and a symmetric probability measure

$$
\mu_{p}=p \delta_{a}+\frac{1-p}{2}\left(\delta_{b}+\delta_{b^{2}}\right) .
$$

Then, for all $p \in(0,1)$, one has $h=\ell v$ [25] but the inequalities are strict in Theorem 1.2. Together with the example of $(\mathbb{Z}<\mathbb{Z} / 3 \mathbb{Z}) \times \mathbb{F}$ (see Example 2.2 above), this shows that equality in Theorem 1.2 and in the fundamental inequality $h \leqslant \ell v$ are independent. 
As far as the free group $\mathbb{F}_{d}=\mathbb{Z} * \cdots * \mathbb{Z}$ is concerned, the above corollary says that the simple random walk is the only symmetric nearest neighbor random walk for which equality holds in Theorem 1.2.

Proof of Corollary 2.5. For $u \in \Sigma=\bigsqcup \Gamma_{i} \backslash\{e\}$, write

$$
\bar{u}=i \quad \text { if } u \in \Gamma_{i} .
$$

A - finite or infinite - word $u_{1} u_{2} \ldots$ over the alphabet $\Sigma$ is reduced if $\overline{u_{i}} \neq \overline{u_{i+1}}$. The group $\Gamma$ is the set of finite reduced words over $\Sigma$ (the identity is the empty word) endowed with the composition law which is the concatenation with possible simplification at the contact point.

Denote by $\mathcal{E}(\Gamma)$ the space of ends of $\Gamma$. Let $\mu$ be a symmetric probability measure on $\Gamma$ with support equal to $S$. Then there exists a unique probability measure $v_{0}$ on $\mathcal{E}(\Gamma)$ which is $\mu$-stationary, and the space $\left(\mathcal{E}(\Gamma), v_{0}\right)$ is (a realization of) the Poisson boundary of $(\Gamma, \mu)$ (see [28, 29] and also [15]). The set $\partial \Gamma$ of right infinite reduced words $\xi=\xi_{1} \xi_{2} \ldots$ over $\Sigma$ is a $\Gamma$-invariant subset of $\mathcal{E}(\Gamma)$ with full $v_{0}$-measure.

For $a \in \Gamma$, denote by

$$
q(a)=\mathbf{P}\left(\exists n, X_{n}=a\right)
$$

the probability that the random walk ever reaches $a$. For $a \in \Sigma$ and $\xi=\xi_{1} \xi_{2} \ldots \epsilon$ $\partial \Gamma$, the Radon-Nikodym derivative

$$
c_{0}(a, \xi)=\frac{\mathrm{d} a_{*}^{-1} v_{0}}{\mathrm{~d} v_{0}}(\xi)
$$

satisfies

$$
c_{0}(a, \xi)= \begin{cases}q(a) & \text { if } \overline{\xi_{1}} \neq \bar{a}, \\ q\left(a \xi_{1}\right) / q\left(\xi_{1}\right) & \text { if } \overline{\xi_{1}}=\bar{a},\end{cases}
$$

see [9], [23] and [25].

Assume that one of the inequalities of Theorem 1.2 is an equality. Proposition 2.4 provides a real number $\alpha \geqslant 0$ such that $c_{0}(a, \xi) \in\left\{e^{\alpha}, e^{-\alpha}\right\}$ for $\mu \otimes v_{0}$-almost every $(a, \xi) \in \Sigma \times \partial \Gamma$. Since $q(a)<1$ as the random walk on a free product different from $\mathbb{Z} / 2 * \mathbb{Z} / 2$ is transient, equation (2.2) implies that $\alpha>0$ and $q(a)=e^{-\alpha}$ for all $a \in S$.

Consider two elements $a, b \in S_{i}$ (possibly with $a=b$ ), with $a b \neq e$. The second case in (2.2) shows that $q(a b) / q(b) \in\left\{e^{\alpha}, e^{-\alpha}\right\}$. Since $q(b)=e^{-\alpha}$, this gives $q(a b) \in\left\{1, e^{-2 \alpha}\right\}$. Since $a b \neq e$ and the random walk is transient, we have $q(a b)<1$, hence $q(a b)=e^{-2 \alpha}$. This gives 


$$
\begin{aligned}
\mathbf{P}\left(\exists m<n, X_{m}=a, X_{n}=a b\right) & =\mathbf{P}\left(\exists m, X_{m}=a\right) \mathbf{P}\left(\exists n, X_{n}=b\right) \\
& =q(a) q(b) \\
& =e^{-2 \alpha} \\
& =q(a b) \\
& =\mathbf{P}\left(\exists n, X_{n}=a b\right),
\end{aligned}
$$

where we used the Markov property for the first equality. This shows that almost every path from $e$ to $a b$ has to pass first through $a$. Equivalently, whenever we write $a b$ as a product $s_{1} \ldots s_{n}$ of elements of $S_{i}$, then some prefix $s_{1} \ldots s_{m}$ is equal to $a$.

This implies that there is no nontrivial loop in the Cayley graph of $\Gamma_{i}$ with respect to $S_{i}$ : if there were such an injective loop

$$
e, a_{1}, a_{1} a_{2}, \ldots, a_{1} a_{2} \ldots a_{k-1}, a_{1} a_{2} \ldots a_{k-1} a_{k}=e
$$

(where all points but the first and last one are distinct), then

$$
a_{1} a_{2}=\left(a_{3} \ldots a_{k}\right)^{-1}=a_{k}^{-1} \ldots a_{3}^{-1} .
$$

Since $S_{i}$ is symmetric, we have written $a_{1} a_{2}$ as a product of elements of $S_{i}$ that never reaches $a_{1}$ (since the loop is injective), a contradiction. This shows that the Cayley graph of $\Gamma_{i}$ with respect to $S_{i}$ is a regular tree, and therefore that $\Gamma_{i}$ is a free product of finitely many factors $\mathbb{Z}$ and $\mathbb{Z} / 2 \mathbb{Z}$.

The Cayley graph of $\Gamma$ with respect to $S$ is also a regular tree. Since the probabilities of ever reaching any neighbor of the origin are the same, so are the transition probabilities, hence $\mu$ is uniform on $\Sigma$.

Corollary 2.5 characterizes the equality case for a class of random walks on free groups, or more generally on some virtually free groups. For hyperbolic groups, this is the only situation where equality in our inequalities is possible:

Corollary 2.6. Let $\Gamma$ be a hyperbolic group which is not virtually free, and let $\mu$ be a finitely supported symmetric probability measure on $\Gamma$ whose support generates $\Gamma$. Then the inequalities of Theorem 1.2 are strict.

Proof. Let $\Gamma$ be a hyperbolic group. If $\mu$ is a finitely supported probability measure on $\Gamma$, then it follows from [1] that the Poisson boundary and the Martin boundary of $(\Gamma, \mu)$ can be identified with $(\partial \Gamma, \nu)$ where $\partial \Gamma$ is the geometric boundary 
of $\Gamma$ and $v$ is the unique $\mu$-stationary measure on $\partial \Gamma$ (it has full support and no atom). In particular, the Martin kernel

$$
c(g, \xi)=\frac{\mathrm{d} g_{*}^{-1} v}{\mathrm{~d} v}(\xi)
$$

is well defined and continuous on $\partial \Gamma$.

Assume that one of the inequalities of Theorem 1.2 is an equality. From Proposition 2.4 , for any $g$ in the support of $\mu$, the continuous function $\xi \mapsto c(g, \xi)$ can only take two values for $\xi$ in the support of $v$, which is the whole space $\partial \Gamma$. Writing any element of the group as a finite product of elements in the support of $\mu$, it follows that $\xi \mapsto c(g, \xi)$ only takes finitely many values, for any $g \in \Gamma$.

Suppose now that $\Gamma$ is not virtually free. It follows that the boundary of $\Gamma$ is not totally disconnected, and moreover the stabilizer of any nontrivial component $L$ of the boundary is a subgroup $\Lambda$ of $\Gamma$, which is quasi-convex and therefore hyperbolic, with limit set equal to $L$ (see the discussion on top of Page 55 in [5] for all these facts). Since $L$ is nontrivial, $\Lambda$ is non-elementary. In particular, it contains an element $g$ of infinite order, which is hyperbolic. The attractive and repulsive points $g^{+}$and $g^{-}$of $g$ both belong to $L$.

The function $\xi \mapsto c(g, \xi)$ is continuous and takes finitely many values. It follows that it is constant on $L$, equal to some $c>0$. It is even equal to $c$ on a small neighborhood $U$ of $L$.

Let $V \subset U$ be a small neighborhood of $g^{+}$. Since $v$ has full support, $v(V)>0$. As $\frac{\mathrm{d} g_{*}^{-1} v}{\mathrm{~d} v}(\xi)=c$ on $V$, we get $c=v(g V) / v(V)$. Iterating $n$ times this equation, we obtain $c^{n}=v\left(g^{n} V\right) / v(V)$. As $g^{n} V$ is attracted to $g^{+}$and $v$ has no atom, we deduce that $c^{n}<1$ for large enough $n$, hence $c<1$. Arguing in the same way using $g^{-1}$ around $g^{-}$, we get $c>1$. This is a contradiction.

\section{Boundaries, and proofs of the main inequalities}

In this section, we prove the two main inequalities of Theorem 1.2. The proof can be equivalently given inside the group (following the ideas of Ledrappier in [22]), or using boundaries. We will mainly use the latter point of view, since it allows for more transparent and intrinsic arguments. Moreover, it gives more insights about the equality case in our inequalities. Nevertheless, in the first subsection, we will quickly sketch the proof inside the group, for the sake of completeness and since it can motivate some definitions on the boundary. 
In this section, $\Gamma$ will always be a countable group with a proper left-invariant distance, and $\mu$ a symmetric probability measure on $\Gamma$ whose support generates $\Gamma$, with finite entropy.

3.1. Proofs inside the group. In this paragraph, we sketch proofs of the inequalities of Theorem 1.2 by arguing inside the group, following Ledrappier [22]. We start with the estimate involving $\ell$.

Let

$$
L(n)=\sum|g| \mu^{* n}(g)
$$

be the average length at time $n$ and

$$
H(n)=-\sum \mu^{* n}(g) \log \mu^{* n}(g)
$$

the entropy at time $n$. Their averages converge respectively to $\ell$ and $h$. If one could compare (a function of) $L(n+1)-L(n)$ with $H(n+1)-H(n)$, an inequality involving $\ell$ and $h$ would follow. It is possible to estimate conveniently those quantities if $\mu(e)>0$ (this is one of the assumptions in [10]) - otherwise, one can replace $\mu$ with $\left(\mu+\delta_{e}\right) / 2$. However, this leads to suboptimal inequalities.

A more efficient procedure, used by Ledrappier [22], is to consider a poissonized version of the random walk, in continuous time, where jumps along the trajectories of the initial random walk occur according to a Poisson distribution. This ensures that, from time $t$ to $t+\varepsilon$, there is a positive probability to stay at the same place, even when $\mu(e)=0$. Formally, define probability measures

$$
\mu_{t}=e^{-t} \sum_{n=0}^{\infty} \frac{t^{n}}{n !} \mu^{* n},
$$

they have the same entropy and drift as the sequence $\mu^{* n}$, i.e.,

$$
H\left(\mu_{t}\right) / t \rightarrow h \quad \text { and } \quad L\left(\mu_{t}\right) / t \rightarrow \ell .
$$

If $P$ denotes the Markov operator associated to $\mu$, one has $\mu_{t}=e^{t(P-I)} \delta_{e}$. Differentiating with respect to $t$, one gets

$$
\mu_{t}^{\prime}(x)=\left((P-I) \mu_{t}\right)(x)=\sum_{g}\left(\mu_{t}(g x)-\mu_{t}(x)\right) \mu(g) .
$$

This gives a formula for the derivative of the entropy:

$$
H\left(\mu_{t}\right)^{\prime}=-\sum_{x, g} \mu(g)\left(\mu_{t}(g x)-\mu_{t}(x)\right)\left(\log \mu_{t}(x)+1\right) .
$$


One would like to use this quantity to dominate functions of the derivative of the drift, but this expression is not convenient to do so since some terms in the sum can be negative, and one should take care of subtle cancellations. Lemma 3 in [22] uses the symmetry of the measure $\mu$ to rewrite the above formula, using a symmetrization procedure, as

$$
H\left(\mu_{t}\right)^{\prime}=\frac{1}{2} \sum_{x, g} \mu(g)\left(\mu_{t}(g x)-\mu_{t}(x)\right)\left(\log \mu_{t}(g x)-\log \mu_{t}(x)\right),
$$

where the terms are all nonnegative.

The derivative of the drift $L\left(\mu_{t}\right)$ is given by

$$
L\left(\mu_{t}\right)^{\prime}=\sum|x| \mu_{t}^{\prime}(x)=\sum_{x, g}|x| \mu(g)\left(\mu_{t}(g x)-\mu_{t}(x)\right) .
$$

It is clear that the derivative of the drift should be bounded by the first moment of the measure, but this is not apparent from this formula. However, using the symmetrization lemma of Ledrappier, one gets

$$
L\left(\mu_{t}\right)^{\prime}=\frac{1}{2} \sum_{x, g}(|x|-|g x|) \mu(g)\left(\mu_{t}(g x)-\mu_{t}(x)\right),
$$

where boundedness becomes more apparent. This formula is more suited to computations. Indeed, let us estimate $|x|-|g x|$ by $|g|$ and let us use Cauchy-Schwarz inequality with respect to the measure $\mu(g) \mu_{t}(x)$ on $\Gamma \times \Gamma$, this yields a bound

$$
\left|L\left(\mu_{t}\right)^{\prime}\right| \leqslant \frac{M_{2}(\mu)}{2}\left(\sum_{x, g} \mu(g) \mu_{t}(x)\left(\frac{\mu_{t}(g x)}{\mu_{t}(x)}-1\right)^{2}\right)^{1 / 2} .
$$

The latter sum has a flavor that is similar to (3.1), that can also be written as

$$
H\left(\mu_{t}\right)^{\prime}=\frac{1}{2} \sum_{x, g} \mu(g) \mu_{t}(x)\left(\frac{\mu_{t}(g x)}{\mu_{t}(x)}-1\right) \log \left(\frac{\mu_{t}(g x)}{\mu_{t}(x)}\right) .
$$

However, it is not possible to compare directly those two quantities using Jensen's inequality: the problem is that the value of $\left(\mu_{t}(g x) / \mu_{t}(x)-1\right)^{2}$ does not determine the value of $\left|\log \left(\mu_{t}(g x) / \mu_{t}(x)\right)\right|$, since the symmetries of those quantities are not the same (additive symmetry around 1 for the former, multiplicative symmetry around 1 for the latter).

The solution to this problem is to estimate (3.2) using Cauchy-Schwarz inequality with respect to a different probability measure on $\Gamma \times \Gamma$, that is more symmetric in a sense, namely $\mu(g) \cdot \frac{\mu_{t}(g x)+\mu_{t}(x)}{2}$. The resulting bound for $L\left(\mu_{t}\right)^{\prime}$ is

$$
\left|L\left(\mu_{t}\right)^{\prime}\right| \leqslant M_{2}(\mu)\left(\sum_{x, g} \mu(g) \frac{\mu_{t}(g x)+\mu_{t}(x)}{2}\left(\frac{\mu_{t}(g x)-\mu_{t}(x)}{\mu_{t}(g x)+\mu_{t}(x)}\right)^{2}\right)^{1 / 2} .
$$


The last factor in this expression can be written as $(c-1)^{2} /(c+1)^{2}$ for $c=\mu_{t}(g x) / \mu_{t}(x)$; it is invariant under the symmetry $c \mapsto c^{-1}$, just like $|\log c|$. It follows that this bound for $L\left(\mu_{t}\right)^{\prime}$ can be compared to $H\left(\mu_{t}\right)^{\prime}$, applying Jensen's inequality to a suitable convex function, with respect again to the probability measure $\mu(g) \cdot \frac{\mu_{t}(g x)+\mu_{t}(x)}{2}$ on $\Gamma \times \Gamma$. The inequality (1.2) follows. The full details will be given later on, in the proof using boundaries.

To prove the estimate involving $\rho$, one uses the function $f_{t}: x \mapsto \mu_{t}(x)^{1 / 2}$ (which has unit norm in $\ell^{2}(\Gamma)$ ). We have $\left\langle P f_{t}, f_{t}\right\rangle \leqslant \rho$ since $\rho$ is the spectral radius of $P$ acting on $\ell^{2}(\Gamma)$. Hence,

$$
\sum_{x, g} \mu(g) \mu_{t}(g x)^{1 / 2} \mu_{t}(x)^{1 / 2} \leqslant \rho .
$$

This expression can not be directly compared to (3.1). One should instead use the (equivalent) inequality $\left\langle(I-P) f_{t}, f_{t}\right\rangle \geqslant 1-\rho$ : here, the scalar product can be again written using the symmetrization lemma, yielding

$$
\frac{1}{2} \sum_{x, g} \mu(g)\left(\mu_{t}(g x)^{1 / 2}-\mu_{t}(x)^{1 / 2}\right)^{2} \geqslant 1-\rho .
$$

Again, this expression has the same flavor as (3.1), and can be compared to it using Jensen's inequality for a good convex function and the probability measure $\mu(g) \cdot \frac{\mu_{t}(g x)+\mu_{t}(x)}{2}$ that ensures the right symmetry of the integrand. This is the only point of the argument where we depart from Ledrappier, who instead relied on the elementary inequality $(a-b)(\log a-\log b) \geqslant 4\left(a^{1 / 2}-b^{1 / 2}\right)^{2}($ Lemma 2 in [22]), which readily gives $1-\rho \leqslant \frac{1}{4} H\left(\mu_{t}\right)^{\prime}$ thanks to (3.3) and (3.1). Again, details will be given later on using boundaries.

In the next sections, we describe the same proofs, but using boundaries. The poissonization procedure will not be needed, and there will be no limit over $t$, all the computations will be direct. This implies that the equality case in our inequalities can be characterized, making it possible to prove Proposition 2.4.

3.2. A symmetrization lemma. It follows from the above proof inside the group that the two crucial points are the symmetrization procedure (Lemma 3 in [22]) that makes it possible to always manipulate nonnegative quantities, and the use of the symmetrized measure $\mu(g) \cdot \frac{\mu_{t}(g x)+\mu_{t}(x)}{2}$ in the inequalities of CauchySchwarz and Jensen. In this subsection, we describe the analogues of those tools in a general $(\Gamma, \mu)$-space. 
Let $(\mathcal{B}, v)$ be a $(\Gamma, \mu)$-space, i.e., a probability space endowed with a $\Gamma$-action for which $v$ is stationary. The Radon-Nikodym cocycle

$$
c(\gamma, \xi)=\frac{\mathrm{d} \gamma_{*}^{-1} v}{\mathrm{~d} v}(\xi)
$$

allows us to define a measure on $\Gamma \times \mathcal{B}$ :

$$
\mathrm{d} m=\frac{c+1}{2} \mathrm{~d} \mu \mathrm{d} \nu .
$$

It is the analogue of $\mu(g) \cdot \frac{\mu_{t}(g x)+\mu_{t}(x)}{2}$. One checks, by means of a change of variables, that $m$ is indeed a probability measure; in fact, for every $\gamma, \frac{c(\gamma, \xi)+1}{2} \mathrm{~d} v(\xi)$ is a probability measure on $\mathcal{B}$. Moreover, since $\mu$ is symmetric, the measure $m$ is invariant under the 'flip' involution $(\gamma, \xi) \mapsto\left(\gamma^{-1}, \gamma \xi\right)$.

The following symmetrization lemma is the analogue of [22, Lemma 3]. The term "symmetrization" comes from the fact that the expression on the right-hand side of (3.4) does not change under the flip involution. It relies crucially on the symmetry of the measure $\mu$. Here and throughout, we will write

$$
d(\gamma, \xi)=\frac{1-c(\gamma, \xi)}{1+c(\gamma, \xi)} \in(-1,1)
$$

Most quantities will be conveniently expressed in terms of $d$. In particular, $c=(1-d) /(1+d)$. If $c$ is replaced by its inverse, then $d$ is replaced by its opposite. Hence, quantities that are invariant under the symmetry $c \mapsto c^{-1}$ give rise to even functions when they are expressed in terms of $d$.

Lemma 3.1. Consider an additive cocycle $f: \Gamma \times \mathcal{B} \rightarrow \mathbb{R}$, i.e., a function satisfying $f\left(\gamma \gamma^{\prime}, \xi\right)=f\left(\gamma, \gamma^{\prime} \xi\right)+f\left(\gamma^{\prime}, \xi\right)$. If $f$ is integrable with respect to $\mathrm{d} \mu \mathrm{d} \nu$, then

$$
\int_{\Gamma \times \mathcal{B}} f(\gamma, \xi) \mathrm{d} \mu(\gamma) \mathrm{d} \nu(\xi)=\int_{\Gamma \times \mathcal{B}} f(\gamma, \xi) d(\gamma, \xi) \mathrm{d} m(\gamma, \xi) .
$$

Proof. This easy computation goes as follows. By the change of variable $g=\gamma^{-1}$ and the symmetry of $\mu$, we have

$$
\int f(\gamma, \xi) \mathrm{d} \mu(\gamma) \mathrm{d} \nu(\xi)=\int f\left(g^{-1}, \xi\right) \mathrm{d} \mu(g) \mathrm{d} \nu(\xi) .
$$

The cocycle relation

$$
f\left(g g^{\prime}, \xi\right)=f\left(g, g^{\prime} \xi\right)+f\left(g^{\prime}, \xi\right)
$$

implies that

$$
f\left(g^{-1}, \xi\right)=-f\left(g, g^{-1} \xi\right) .
$$


The change of variable $\eta=g^{-1} \xi$ gives

$$
\int f \mathrm{~d} \mu \mathrm{d} v=-\int f(g, \eta) \mathrm{d}\left(g_{*}^{-1} v\right)(\eta) \mathrm{d} \mu(g)=\int f \frac{-2 c}{1+c} \mathrm{~d} m .
$$

On the other hand, we have of course

$$
\int f \mathrm{~d} \mu \mathrm{d} \nu=\int f \frac{2}{1+c} \mathrm{~d} m .
$$

The half-sum of these two relations gives the desired result.

3.3. The Poisson boundary, proof of the first main inequality. In this paragraph, we prove the first inequality (1.1) of our main theorem, relating $\rho$ and $h$. The proof relies on the action of $(\Gamma, \mu)$ on its Poisson boundary $\left(\mathcal{B}_{0}, v_{0}\right)$, that we described quickly in Section 2 (see $[19,11]$ for more details). Let $c_{0}, d_{0}$ and $m_{0}$ be the objects defined above, attached to the Poisson boundary.

Kaimanovich and Vershik [19] proved the following formula for the entropy:

$$
h=-\int_{\Gamma \times \mathcal{B}_{0}} \log c_{0} \mathrm{~d} \mu \mathrm{d} v_{0} .
$$

Since the Radon-Nikodym derivative $c_{0}$ is a multiplicative cocycle, the symmetrization lemma 3.1 applies:

$$
h=-\int_{\Gamma \times \mathcal{B}_{0}} \log c_{0} \cdot d_{0} \mathrm{~d} m_{0} .
$$

We have $c_{0}=\frac{1-d_{0}}{1+d_{0}}$ by definition of $d_{0}$. Hence, writing

$$
F(x)=2 x \operatorname{artanh}(x)=x \log \left(\frac{1+x}{1-x}\right)=\sum_{n \geqslant 1} \frac{2}{2 n-1} x^{2 n},
$$

we get the following expression for the entropy:

$$
h=\int_{\Gamma \times \mathcal{B}_{0}} F\left(d_{0}\right) \mathrm{d} m_{0} .
$$

Note that the function $F$ is even.

We will now get a bound from below for the spectral radius of the random walk, using an object living on the Poisson boundary. 
Lemma 3.2. One has

$$
\rho \geqslant \int_{\Gamma \times \mathcal{B}_{0}} c_{0}^{1 / 2} \mathrm{~d} \mu \mathrm{d} \nu_{0}
$$

Proof. Let us define a function $f_{n}$ on $\Gamma$ by $f_{n}(x)=\mu^{* n}(x)^{1 / 2}$. It has unit norm in $L^{2}$. Denoting by $P$ the Markov operator associated to the random walk, we get

$$
\begin{aligned}
\rho & \geqslant\left\langle P f_{n-1}, f_{n}\right\rangle \\
& =\sum_{x} \sum_{g} \mu(g) f_{n-1}(x g) f_{n}(x) \\
& =\sum_{y} \sum_{g} \mu(g) f_{n-1}\left(g^{-1} y\right) f_{n}(y) \\
& =\sum_{y} \sum_{g} \mu(g)\left(\frac{\mu^{* n-1}\left(g^{-1} y\right)}{\mu^{* n}(y)}\right)^{1 / 2} \mu^{* n}(y) .
\end{aligned}
$$

Let $\mathbf{P}$ denote the probability distribution of the random walk on the space $\Omega$ of trajectories starting from the identity. Write $\omega_{n}$ for the position at time $n$ of a trajectory, $\mathcal{F}_{n}$ for the $\sigma$-algebra generated by $\omega_{n}, \omega_{n+1}, \ldots$ and $C_{g}$ for the set of trajectories with $\omega_{1}=g$. Then

$$
\frac{\mu(g) \mu^{* n-1}\left(g^{-1} \omega_{n}\right)}{\mu^{* n}\left(\omega_{n}\right)}=\mathbf{P}\left(C_{g} \mid \mathcal{F}_{n}\right)(\omega) .
$$

This converges almost surely to $\mathbf{P}\left(C_{g} \mid \mathcal{F}_{\infty}\right)(\omega)$, where $\mathcal{F}_{\infty}=\bigcap \mathcal{F}_{n}$ is the tail $\sigma$-algebra. The Poisson boundary $\left(\mathcal{B}_{0}, \nu_{0}\right)$ is the quotient of $(\Omega, \mathbf{P})$ by $\mathcal{F}_{\infty}$. Denoting by bnd: $\Omega \rightarrow \mathcal{B}_{0}$ the quotient map, we deduce that $\mu^{* n-1}\left(g^{-1} \omega_{n}\right) / \mu^{* n}\left(\omega_{n}\right)$ converges almost surely to a function of bnd $(\omega)$, which is in fact $c_{0}(g$, bnd $(\omega))$ (see [15, Paragraph 3.2]). This function is bounded from above (by $\left.\mu(g)^{-1}\right)$, hence convergence in $L^{1}$ follows. We obtain

$$
\begin{aligned}
\sum_{y}\left(\frac{\mu^{* n-1}\left(g^{-1} y\right)}{\mu^{* n}(y)}\right)^{1 / 2} \mu^{* n}(y) & =\int_{\Omega}\left(\mu(g)^{-1} \mathbf{P}\left(C_{g} \mid \mathcal{F}_{n}\right)(\omega)\right)^{1 / 2} \mathrm{~d} \mathbf{P}(\omega) \\
& \longrightarrow \int_{\Omega} c_{0}(g, \operatorname{bnd}(\omega))^{1 / 2} \mathrm{~d} \mathbf{P}(\omega) \\
& =\int_{\mathcal{B}_{0}} c_{0}(g, \xi)^{1 / 2} \mathrm{~d} \nu_{0}(\xi) .
\end{aligned}
$$

The result follows from this convergence and (3.9). 
Remark 3.3. A "more abstract" proof of Lemma 3.2 can also be obtained by using the fact that since the action of the group $\Gamma$ on the Poisson boundary $\left(\mathcal{B}_{0}, v_{0}\right)$ is amenable [31, Corollary 5.3], the associated quasi-regular representation $\pi$ of $\Gamma$ is weakly contained in the regular representation $\pi_{\text {reg }}$ [20]. Denote by $\pi_{\text {reg }}(\mu)$ and $\pi(\mu)$ the averages of $\pi_{\text {reg }}$ and $\pi$ with respect to $\mu$. Now, the right-hand side of inequality 3.8 is precisely $\langle\pi(\mu) 1,1\rangle$, whereas by the above weak containment property $\|\pi(\mu)\| \leq\left\|\pi_{\text {reg }}(\mu)\right\|=\rho$.

Since $\mathrm{d} m_{0}=\frac{c_{0}+1}{2} \mathrm{~d} \mu \mathrm{d} v_{0}$, the integral in (3.8) is equal to

$$
\int_{\Gamma \times \mathcal{B}_{0}} \frac{2 c_{0}^{1 / 2}}{1+c_{0}} \mathrm{~d} m_{0}
$$

We rewrite this expression in terms of $d_{0}$ : since $c_{0}=\left(1-d_{0}\right) /\left(1+d_{0}\right)$, we have

$$
2 c_{0}^{1 / 2} \cdot \frac{1}{1+c_{0}}=2\left(\frac{1-d_{0}}{1+d_{0}}\right)^{1 / 2} \cdot \frac{1}{2 /\left(1+d_{0}\right)}=\left(1-d_{0}^{2}\right)^{1 / 2} .
$$

Therefore,

$$
1-\rho \leqslant 1-\int_{\Gamma \times \mathcal{B}_{0}} \frac{2 c_{0}^{1 / 2}}{1+c_{0}} \mathrm{~d} m_{0}=\int_{\Gamma \times \mathcal{B}_{0}} G\left(d_{0}\right) \mathrm{d} m_{0},
$$

where $G(x)=1-\left(1-x^{2}\right)^{1 / 2}$. This function is even on $[-1,1]$, its restriction to $[0,1]$ is an increasing bijection of $[0,1]$.

Lemma 3.4. The function $F \circ G^{-1}$ satisfies on $[0,1)$

$$
F \circ G^{-1}(x)=\left(2 x-x^{2}\right)^{1 / 2} \log \left(\frac{1+\left(2 x-x^{2}\right)^{1 / 2}}{1-\left(2 x-x^{2}\right)^{1 / 2}}\right)=\sum_{n=1}^{\infty} c_{n} x^{n},
$$

where $c_{1}=4$ and $(2 n-1) c_{n}=(n-2) c_{n-1}+2$ for $n \geqslant 2$. In particular, the coefficients $c_{n}$ are positive. Hence, $F \circ G^{-1}$ is increasing and convex.

Proof. A simple computation shows that the function $H=F \circ G^{-1}$ satisfies the differential equation

$$
H^{\prime}(x)=\frac{1-x}{x(2-x)} H(x)+\frac{2}{1-x} .
$$

Multiplying by $x(2-x)$ and identifying the Taylor coefficients on the left and on the right, one gets the recurrence relation $(2 n-1) c_{n}=(n-2) c_{n-1}+2$ for $n \geqslant 2$. 
The map $F \circ G^{-1}$ is increasing, so the inequality (3.10) transforms into

$$
F \circ G^{-1}(1-\rho) \leqslant F \circ G^{-1}\left(\int_{\Gamma \times \mathcal{B}_{0}} G\left(d_{0}\right) \mathrm{d} m_{0}\right) .
$$

Note that the partial inverse $G^{-1}$ of $G$ satisfies $F \circ G^{-1} \circ G=F$ on the interval $(-1,1)$, because both $F$ and $G$ are even functions. Since $F \circ G^{-1}$ is convex by Lemma 3.4, Jensen's inequality implies that

$$
F \circ G^{-1}(1-\rho) \leqslant \int_{\Gamma \times \mathcal{B}_{0}} F\left(d_{0}\right) \mathrm{d} m_{0} .
$$

Thanks to (3.7), this proves $(1.1)$ since $G^{-1}(1-\rho)=\sqrt{1-\rho^{2}}$.

\subsection{The Busemann compactification, proof of the second main inequality.} For the proof of the second inequality (1.2) of our main theorem, relating $\ell$ and $h$, we will need another more geometric boundary, which will give us access to the metric notion of linear drift, in contrast to the Poisson boundary which is purely a measure theoretic construction.

We recall the construction of the Busemann (horospherical) closure of the group $\Gamma$. It is obtained by embedding $\Gamma$ into Lipschitz functions on $\Gamma$ using the distance kernel, as follows. Let $X \subset \mathbb{R}^{\Gamma}$ be the set of 1-Lipschitz real-valued functions on $\Gamma$ which vanish on $e$. Lipschitz means here that $\left|\varphi\left(g g^{\prime}\right)-\varphi(g)\right| \leqslant\left|g^{\prime}\right|$. For any $\gamma \in \Gamma$,

$$
\Phi_{\gamma}(g)=\left|\gamma^{-1} g\right|-\left|\gamma^{-1}\right|
$$

defines an element of $X$, and the assignment $\gamma \mapsto \Phi_{\gamma}$ is continuous, injective. Let $\mathcal{B}_{1}$ be the closure of the image of $\Gamma$. The action of $\Gamma$ on $\mathcal{B}_{1}$ is given by

$$
(\gamma \xi)(g)=\xi\left(\gamma^{-1} g\right)-\xi\left(\gamma^{-1}\right) .
$$

The latter equation for the action is better understood if one thinks of $X$ as the quotient set of 1-Lipschitz functions on $\Gamma$ modulo the constants, endowed with the natural translation action on functions. Each element of $X$ has a unique representative which vanishes at $e$, which explains the above formula.

Karlsson and Ledrappier [17] and [18, Theorem 18] proved that in this setting, under the assumption of finite first moment, there exists an ergodic stationary probability measure $v_{1}$ on $\mathcal{B}_{1}$ satisfying

$$
\ell=\int_{\Gamma \times \mathcal{B}_{1}} \xi\left(\gamma^{-1}\right) \mathrm{d} \mu(\gamma) \mathrm{d} \nu_{1}(\xi) .
$$


Karlsson and Ledrappier [18] call this expression for $\ell$ a Furstenberg-Khasminskii formula.

By definition of the action, the assignment $\beta:(g, \xi) \mapsto \xi\left(g^{-1}\right)$ satisfies

$$
\beta\left(g g^{\prime}, \xi\right)=\xi\left(g^{\prime-1} g^{-1}\right)=\left(g^{\prime} \xi\right)\left(g^{-1}\right)+\xi\left(g^{\prime-1}\right)=\beta\left(g, g^{\prime} \xi\right)+\beta\left(g^{\prime}, \xi\right),
$$

so it is an additive cocycle; this is in fact the classical Busemann cocycle. Hence, the symmetrization Lemma 3.1 applies, and we find

$$
\ell=\int_{\Gamma \times \mathcal{B}_{1}} \beta \cdot d_{1} \mathrm{~d} m_{1},
$$

where $d_{1}=\left(1-c_{1}\right) /\left(1+c_{1}\right)$ with $c_{1}$ the Radon-Nikodym derivative, and $m_{1}=\frac{1+c_{1}}{2} \mathrm{~d} \mu \mathrm{d} v_{1}$.

Kaimanovich and Vershik [19] proved that the boundary entropy of a $(\Gamma, \mu)$-space is always less than or equal to the entropy of the random walk. Applying this result to $\left(\mathcal{B}_{1}, v_{1}\right)$, we get $-\int_{\Gamma \times \mathcal{B}_{1}} \log c_{1} \mathrm{~d} \mu \mathrm{d} \nu_{1} \leqslant h$. The left-hand side can be transformed using the symmetrization lemma 3.1, giving

$$
\int_{\Gamma \times \mathcal{B}_{1}} F\left(d_{1}\right) \mathrm{d} m_{1} \leqslant h .
$$

We can now prove our second main inequality (1.2) comparing $\ell$ and $h$. We start from (3.12) and apply Cauchy-Schwarz inequality, yielding

$$
\ell \leqslant\left(\int_{\Gamma \times \mathcal{B}_{1}}|\beta|^{2} \mathrm{~d} m_{1}\right)^{1 / 2}\left(\int_{\Gamma \times \mathcal{B}_{1}} d_{1}^{2} \mathrm{~d} m_{1}\right)^{1 / 2} .
$$

Since $|\beta(g, \xi)| \leqslant|g|$, because $\mathcal{B}_{1}$ consists of 1-Lipschitz functions vanishing at $e$, the first factor on the right-hand side is bounded by $M_{2}(\mu)$. Writing $\tilde{\ell}=\ell / M_{2}(\mu)$, we obtain

$$
\tilde{\ell}^{2} \leqslant \int_{\Gamma \times \mathcal{B}_{1}} d_{1}^{2} \mathrm{~d} m_{1}
$$

It follows from the Taylor expansion of the function $F$, given in (3.6), that $\tilde{F}(x)=$ $F\left(x^{1 / 2}\right)$ is convex on $[0,1)$. Applying $\tilde{F}$ to the previous inequality and using Jensen inequality, we get

$$
F(\tilde{\ell}) \leqslant \int_{\Gamma \times \mathcal{B}_{1}} F\left(d_{1}\right) \mathrm{d} m_{1} .
$$

By (3.13), the right-hand side is bounded by $h$. This proves (1.2).

The above proof can be refined, to get a slightly stronger inequality. For any $p \geqslant 1$, let $M_{p}(\mu)=\left(\sum_{g}|g|^{p} \mu(p)\right)^{1 / p}$ be the $\ell^{p}$-norm with respect to the measure $\mu$ of the distance to the identity, generalizing the notation $M_{2}(\mu)$. 
Proposition 3.5. Let $\mu$ be a symmetric probability measure with finite first moment on a countable group $\Gamma$ with a proper left-invariant distance. Then

$$
\sum_{n=1}^{\infty} \frac{2}{2 n-1}\left(\frac{\ell}{M_{1+1 /(2 n-1)}(\mu)}\right)^{2 n} \leqslant h .
$$

In this estimate, the first terms of the expansion vanish if the corresponding moments are infinite. This proposition gives a nontrivial estimate when $\mu$ has a finite moment of some order $p>1$. In particular, if $\mu$ has a moment of order $1+1 /(2 n-1)$, we get

$$
\ell \leqslant M_{1+1 /(2 n-1)}(\mu)\left(\frac{2 n-1}{2} h\right)^{1 /(2 n)} .
$$

If $h=0$ for such a measure, it follows that $\ell=0$. This is a weak version of a theorem of Karlsson and Ledrappier [17], stating that this implication holds for symmetric measures with a finite moment of order 1 (the symmetry assumption can even be replaced by a weaker centering assumption).

Note that, since $M_{p}(\mu) \leqslant M_{2}(\mu)$ for $p \leqslant 2$ and $F(x)=\sum \frac{2}{2 n-1} x^{2 n}$, this proposition strengthens the inequality (1.2).

Proof of Proposition 3.5. Let $n \geqslant 1$ be an integer. We start again from (3.12), but we use Hölder inequality for the exponent $1+1 /(2 n-1)$ and the conjugate exponent $2 n$ : it follows that the drift $\ell$ satisfies

$$
\ell \leqslant\left(\int_{\Gamma \times \mathcal{B}_{1}}|\beta|^{1+1 /(2 n-1)} \mathrm{d} m_{1}\right)^{(2 n-1) /(2 n)}\left(\int_{\Gamma \times \mathcal{B}_{1}} d_{1}^{2 n} \mathrm{~d} m_{1}\right)^{1 /(2 n)} .
$$

Since $|\beta(g, \xi)| \leqslant|g|$, the first factor is bounded by $M_{1+1 /(2 n-1)}(\mu)$. Thus,

$$
\left(\frac{\ell}{M_{1+1 /(2 n-1)}(\mu)}\right)^{2 n} \leqslant \int_{\Gamma \times \mathcal{B}_{1}} d_{1}^{2 n} \mathrm{~d} m_{1} .
$$

Note that the previous equation makes sense even if $\mu$ has no finite moment of order $1+1 /(2 n-1)$ (in this case, the left-hand size vanishes, and the equation is trivial).

Multiplying this inequality by $2 /(2 n-1)$ and summing over $n$, we obtain

$$
\begin{aligned}
\sum_{n \geqslant 1} \frac{2}{2 n-1}\left(\frac{\ell}{M_{1+1 /(2 n-1)}(\mu)}\right)^{2 n} & \leqslant \int_{\Gamma \times \mathcal{B}_{1}} \sum_{n \geqslant 1} \frac{2}{2 n-1} d_{1}^{2 n} \mathrm{~d} m_{1} \\
& =\int_{\Gamma \times \mathcal{B}_{1}} F\left(d_{1}\right) \mathrm{d} m_{1} .
\end{aligned}
$$

By (3.13), this is at most $h$. 
3.5. Discussion of the equality case. The proofs given in the previous paragraphs imply that the equality situation in those inequalities is very rigid. We can use this information to prove Proposition 2.4.

Proof of Proposition 2.4. Assume first that the inequality (1.1) comparing $\rho$ and $h$ is an equality. Then all the inequalities in the proof of this inequality have to be equalities. In particular, Jensen's inequality after (3.11) is an equality, whence $G\left(d_{0}\right)$ is almost surely constant, i.e., there exists $a \in \mathbb{R}$ such that $d_{0}= \pm a$ almost surely. Since $c_{0}=\left(1-d_{0}\right) /\left(1+d_{0}\right)$, it follows that $c_{0}$ almost surely takes the values $(1-a) /(1+a)$ or $(1+a) /(1-a)$, which are inverse of each other.

Assume now that the inequality (1.2) comparing $\ell$ and $h$ is an equality. Denote by $\left(\mathcal{B}_{1}, v_{1}\right)$ the Busemann compactification used in Paragraph 3.4. We have the inequalities

$$
\begin{aligned}
F\left(\frac{\ell}{M_{2}(\mu)}\right) & =\sum \frac{2}{2 n-1}\left(\frac{\ell}{M_{2}(\mu)}\right)^{2 n} \\
& \leqslant \sum \frac{2}{2 n-1}\left(\frac{\ell}{M_{1+1 /(2 n-1)}(\mu)}\right)^{2 n} \\
& \leqslant \int_{\Gamma \times \mathcal{B}_{1}} F\left(d_{1}\right) \mathrm{d} m_{1} \\
& =-\int_{\Gamma \times \mathcal{B}_{1}} \log c_{1} \mathrm{~d} \mu \mathrm{d} \nu_{1} \leqslant h .
\end{aligned}
$$

If the extreme terms are equal, we have equality everywhere.

All the moments of $\mu$ coincide, hence $\mu$ is supported on points at a fixed distance of $e$. There must also be equality $m_{1}$-almost everywhere in the inequality $|\beta(g, \xi)| \leqslant|g|$ that we used just after (3.14). This implies that $|\beta|$ is almost surely constant. Finally, there is equality in the Cauchy-Schwarz inequality (3.14), hence, $d_{1}$ is almost surely proportional to $\beta$. It follows that $\left|d_{1}\right|$ is almost surely constant. Hence, as in the first case, $c_{1}$ takes only two values which are inverse of each other. To conclude, we should prove that this property (that we have proved on $\left.\left(\mathcal{B}_{1}, v_{1}\right)\right)$ also holds on the Poisson boundary, since the statement of the proposition is formulated on the Poisson boundary.

Since equality holds everywhere in (3.15), one has in particular

$$
-\int_{\Gamma \times \mathcal{B}_{1}} \log c_{1} \mathrm{~d} \mu \mathrm{d} \nu_{1}=h,
$$

i.e., the entropy of the $(\Gamma, \mu)$-space $\left(\mathcal{B}_{1}, v_{1}\right)$ is maximal. By [19, Theorem 3.2], this implies that $\left(\mathcal{B}_{1}, v_{1}\right)$ is the Poisson boundary if the Radon-Nikodym cocycle 
separates the points, i.e., if for almost every points $\xi \neq \eta$ there exists $g \in \Gamma$ such that $c_{1}(g, \xi) \neq c_{1}(g, \eta)$. In general, the Poisson boundary is a factor of $\left(\mathcal{B}_{1}, v_{1}\right)$, obtained by identifying the points that are not separated by the Radon-Nikodym cocycle. In particular, any property of the Radon-Nikodym cocycle that is true on $\left(\mathcal{B}_{1}, v_{1}\right)$ is also true on the Poisson boundary. This concludes the proof.

\section{References}

[1] A. Ancona, Positive harmonic functions and hyperbolicity. In J. Král, J. Lukeš, I. Netuka, and J. Veselý, Potential theory. Surveys and problems. (Prague, 1987.) Lecture Notes in Mathematics, 1344. Springer-Verlag, Berlin, 1988, 1-23. Zbl 0677.31006 MR 973878

[2] A. Avez, Croissance des groupes de type fini et fonctions harmoniques. In J.-P. Conze and M. S. Keane (eds.), Théorie ergodique. Théorie ergodique (Actes Journées Ergodiques, Rennes, 1973/1974). Lecture Notes in Mathematics, 532. Springer, Berlin, 1976, 35-49. Zbl 0482911 MR 0482911

[3] L. Bartholdi, Cactus trees and lower bounds on the spectral radius of vertex-transitive graphs. In V. A. Kaimanovich, in collaboration with K. Schmidt and W. Woess (eds.), Random walks and geometry. (Vienna 2011). de Gruyter, Berlin, 2004, 349-361. Zbl 1103.60048 MR 2087788

[4] S. Blachère, P. Haïssinsky, and P. Mathieu, Harmonic measures versus quasiconformal measures for hyperbolic groups. Ann. Sci. Éc. Norm. Supér. (4) 44 (2011), 683-721. Zbl 1243.60005 MR 2919980

[5] B. H. Bowditch, Boundaries of strongly accessible hyperbolic groups. In I. Rivin, C. Rourke and C. Series (eds.) The Epstein birthday schrift. Geometry \& Topology Monographs, 1. Geometry \& Topology Publications, Coventry, 1998, 51-97. Zbl 0918.20027 MR 1668331

[6] Th. K. Carne, A transmutation formula for Markov chains. Bull. Sci. Math. (2) 109 (1985), 399-405. Zbl 0584.60078 MR 837740

[7] Y. Derriennic, Entropie, théorèmes limite et marches aléatoires. In H. Heyer. (ed.), Probability measures on groups. VIII. (Oberwolfach, 1985.) Lecture Notes in Mathematics, 1210. Springer, Berlin, 1986, 241-284. Zbl 0612.60005 MR 879010

[8] P. de la Harpe, Topics in geometric group theory. Chicago Lectures in Mathematics. University of Chicago Press, Chicago, IL, 2000. Zbl 0965.20025 MR 1786869

[9] E. B. Dynkin and M. B. Maljutov, Random walk on groups with a finite number of generators. Dokl. Akad. Nauk SSSR 137 (1961), 1042-1045. In Russian. English transl., Soviet Math. Dokl. 2 (1961), 399-402. Zbl 0214.44101 MR 0131904

[10] A. Erschler and A. Karlsson, Homomorphisms to $\mathbb{R}$ constructed from random walks. Ann. Inst. Fourier (Grenoble) 60 (2010), 2095-2113. Zbl 1274.60015 MR 2791651 
[11] A. Furman, Random walks on groups and random transformations. In B. Hasselblatt and A. Katok (eds.), Handbook of dynamical systems. Vol. 1A. North-Holland, Amsterdam, 2002, 931-1014. Zbl 1053.60045 MR 1928529

[12] Y. Guivarc'h, Sur la loi des grands nombres et le rayon spectral d'une marche aléatoire. In Journées sur les Marches Aléatoires. (Kleebach, 1979.) Astérisque, 74. Société Mathématique de France, Paris, 1980, 47-98. Zbl 0448.60007 MR 588157

[13] V. A. Kaŭmanovich, Brownian motion and harmonic functions on covering manifolds. An entropic approach. Dokl. Akad. Nauk SSSR 288 (1986), 1045-1049. In Russian. English transl., Soviet Math. Dokl. 33 (1986), no. 3, 812-816. Zbl 0615.60074 MR 852647

[14] V. A. Kaĭmanovich, The Poisson formula for groups with hyperbolic properties. Ann. of Math. (2) 152 (2000), no. 3, 659-692. Preprint 1998. arXiv:math/9802132v1 [math.DS]

[15] V. A. Kaĭmanovich, The Poisson formula for groups with hyperbolic properties. Ann. of Math. (2) 152 (2000), 659-692. Zbl 0984.60088 MR 1815698

[16] H. Kesten, Symmetric random walks on groups. Trans. Amer. Math. Soc. 92 (1959), 336-354. Zbl 0092.33503 MR 0109367

[17] A. Karlsson and F. Ledrappier, Linear drift and Poisson boundary for random walks. Pure Appl. Math. Q. 3 (2007), 1027-1036. Zbl 1142.60035 MR 2402595

[18] A. Karlsson and F. Ledrappier, Noncommutative ergodic theorems. In B. Farb and D. Fisher (eds.), Geometry, rigidity, and group actions. Chicago Lectures in Mathematics. University of Chicago Press, Chicago, IL, 2011, 396-418. Zbl 06077153 MR 2807838

[19] V. A. Kaĭmanovich and A. M. Vershik, Random walks on discrete groups: boundary and entropy. Ann. Probab. 11 (1983), 457-490. Zbl MR 704539

[20] M. G. Kuhn, Amenable actions and weak containment of certain representations of discrete groups. Proc. Amer. Math. Soc. 122 (1994), 751-757. Zbl 0829.43003 MR 1209424

[21] F. Ledrappier, Harmonic measures and Bowen-Margulis measures. Israel J. Math. 71 (1990), 275-287. Zbl 0728.53029 MR 1088820

[22] F. Ledrappier, Sharp estimates for the entropy. In M. A. Picardello (ed.), Harmonic analysis and discrete potential theory. (Frascati, 1991.) Plenum Press, New York, 1992, 281-288. MR 1222466

[23] F. Ledrappier, Some asymptotic properties of random walks on free groups. In J. C. Taylor (ed.), Topics in probability and Lie groups: boundary theory. CRM Proceedings \& Lecture Notes, 28. American Mathematical Society, Providence, R.I., 2001. 117-152. Zbl 0994.60073 MR 1832436

[24] F. Ledrappier, Linear drift and entropy for regular covers. Geom. Funct. Anal. 20 (2010), 710-725. Zbl 1208.58033 MR 2720229

[25] J. Mairesse and F. Mathéus, Random walks on free products of cyclic groups. J. Lond. Math. Soc. (2) 75 (2007), 47-66. Zbl 1132.60054 MR 2302729 
[26] T. Nagnibeda, An estimate from above of spectral radii of random walks on surface groups. Zap. Nauchn. Sem. S.-Peterburg. Otdel. Mat. Inst. Steklov. (POMI) 240 (1997), Teor. Predst. Din. Sist. Komb. i Algoritm. Metody. 2, 154-165, 293-294. In Russian. English transl., J. Math. Sci. (New York) 96 (1999), no. 5, 3542-3549. Zbl 0947.60006 MR 1691645

[27] N. Th. Varopoulos, Long range estimates for Markov chains. Bull. Sci. Math. (2) 109 (1985), 225-252. Zbl 0583.60063 MR 822826

[28] W. Woess, Boundaries of random walks on graphs and groups with infinitely many ends. Israel J. Math. 68 (1989), 271-301. Zbl 0723.60009 MR 1039474

[29] W. Woess, Fixed sets and free subgroups of groups acting on metric spaces. Math. Z. 214 (1993), 425-439. Zb1 0892.54022 MR 1245204

[30] W. Woess, Random walks on infinite graphs and groups. Cambridge Tracts in Mathematics, 138. Cambridge University Press, Cambridge, 2000. Zbl 0951.60002 MR 1743100

[31] R. J. Zimmer, Amenable ergodic group actions and an application to Poisson boundaries of random walks. J. Functional Analysis 27 (1978), 350-372. Zbl 0391.28011 MR 0473096

Received May 22, 2013

Sébastien Gouëzel, IRMAR, Université Rennes 1, 35042 Rennes Cedex, France e-mail: sebastien.gouezel@univ-rennes1.fr

Frédéric Mathéus, Université de Bretagne Sud, L.M.B.A., UMR 6205, BP 573, 56017 Vannes, France

e-mail: Frederic.Matheus@univ-ubs.fr

François Maucourant, IRMAR, Université Rennes 1, 35042 Rennes Cedex, France e-mail: francois.maucourant@univ-rennes1.fr 